\title{
Transient Neonatal Diabetes Mellitus Managed with Continuous Subcutaneous Insulin Infusion (CSII) and Continuous Glucose Monitoring
}

Min Soo Kim, MD, Sung Eun Kim, MD, Na Yeong Lee, MD, Seul Ki Kim, MD, Shin Hee Kim, MD, Won Kyoung Cho, MD, PhD, Kyoung Soon Cho, MD, Min Ho Jung, MD, PhD, Byung-Kyu Suh, MD, PhD, and Moon Bae Ahn, $\mathrm{MD}$

Department of Pediatrics, College of Medicine, The Catholic University of Korea, Seoul, Korea

\section{ABSTRACT}

Neonatal diabetes mellitus can be categorized as transient, permanent, or syndromic, and approximately half of the cases are transient. We present a case involving a term newborn who showed overt progression of transient neonatal diabetes mellitus, with complete remission within 6 months. On the second day of life, the patient presented with tachypnea, hyperglycemia, and decreased serum levels of C-peptide and insulin. Continuous subcutaneous infusion of insulin and continuous glucose monitoring were well tolerated. The patient showed a normal growth pattern, with no hyperglycemic or hypoglycemic episodes at 6 months of age. As it is rare and often asymptomatic, hyperglycemia may be attributed to various factors, including intrauterine environment, perinatal stress, and diverse genetic background. Therefore, consistent blood glucose monitoring and prompt early insulin therapy are crucial for any term newborns with persistent hyperglycemia, to prevent further diabetic complications. Moreover, continuous subcutaneous insulin infusion and the utilization of continuous glucose monitoring devices are the most effective and practical management strategies.

Key Words: Diabetes mellitus, transient neonatal; Insulin infusion systems; Blood glucose self-monitoring; Diabetes mellitus; Infant, newborn

\section{INTRODUCTION}

Neonatal diabetes mellitus (NDM) is a rare form of monogenic diabetes that is diagnosed within the first 6 months of life. It occurs in approximately 1:90,000 to 160,000 births, with at least 20 causative genes ${ }^{1}$. NDM can be transient or permanent. Transient neonatal diabetes mellitus (TNDM), which accounts for approximately half of the total NDM cases, usually resolves within 3 months of insulin therapy; however, it may recur as permanent diabetes
Received: 11 September 2020

Revised: 13 October 2020

Accepted: 15 October 2020

Correspondence to: Moon Bae Ahn, $\mathrm{MD}$

Department of Pediatrics, College of Medicine, The Catholic University of Korea, 222 Banpo-daero, Seocho-gu, Seoul 06591, Korea

Tel: +82-2-2258-6756

Fax: +82-2-537-4544

E-mail:mbahn@catholic.ac.kr

Copyright(c)

By Korean Society of Neonatology.

All right reserved.

This is an Open-Access article distributed under the terms of the Creative Commons At tribution Non-Commercial License (http:// creativecommons.org/licenses/by-nc/4.0), which permits unrestricted non-commercial use, distribution, and reproduction in any medium, provided the original work is pro perly cited. 
later in life. Conversely, permanent neonatal diabetes mellitus (PNDM) requires lifelong insulin therapy ${ }^{2}$. TNDM is frequently caused by the overexpression of PLAGL1 and HYMA1 due to defects in 6q24, and the overall prevalence and presenting features vary with race and ethnicity ${ }^{3)}$. More than $40 \%$ of PNDM cases are caused by mutations in either $K C N J 11$ or $A B C C 8^{4)}$.

Continuous subcutaneous insulin infusion (CSII) and realtime continuous glucose monitoring (CGM) are advanced technologies that could be applied to any young patients with diabetes. Currently, CSII is the treatment of choice for the initial management of NDM because it allows the delivery of very small, accurate doses of insulin ${ }^{1)}$. The utilization of a CGM device together with CSII reduces the need for frequent blood puncture and provides a minimally invasive method of 24-hour glucose monitoring.

Early genetic diagnosis and early management of NDM are crucial in understanding the clinical course and long-term outcome, as they affect the therapeutic decisions. However, most of the mutations causing NDM are rare recessive, which hinders their identification, and the genetic diagnosis of approximately $20 \%$ of NDM cases is still unknown ${ }^{1)}$. Herein, we present a case involving an infant with overt progression of TNDM during the early postnatal period, managed with CSII and CGM.

\section{CASE REPORT}

A male infant, the only child of a nonconsanguineous Korean couple, was vaginally delivered at 38 weeks of gestation at a local hospital, with a birth weight of $2.7 \mathrm{~kg}$ (3rd to 10th centile) and a length of $49.0 \mathrm{~cm}$ (10th to 50th centile) $)^{5}$. Dysmorphic features were not observed. No family history of medical conditions, including diabetes mellitus, was declared. On day 2 , he was referred to the neonatal intensive care unit (NICU) because of tachypnea (breath rate, 70/min) and hyperglycemia (blood glu cose level, $381 \mathrm{mg} / \mathrm{dL}$ ). His systolic and diastolic blood pressures were 74 and $42 \mathrm{~mm} \mathrm{Hg}$, respectively. His heart rate was 112 beats/ min, and his body temperature was $36.4^{\circ} \mathrm{C}$. His respiration rate normalized within 48 hours of admission to the NICU; however, he remained hyperglycemic (blood glucose level, 290 to 390 $\mathrm{mg} / \mathrm{dL})$. His serum C-peptide and insulin levels were $0.06 \mathrm{ng} /$ $\mathrm{mL}$ and undetectable $(<0.20 \mu \mathrm{U} / \mathrm{mL})$, respectively (Table 1$)$. His baseline chemistry, including serum blood urea nitrogen (15.3 $\mathrm{mg} / \mathrm{dL}$; normal, 7.0 to 20.0 ), creatinine $(0.9 \mathrm{mg} / \mathrm{dL}$; normal, 0.6 to 1.2), aspartate aminotransferase (38 U/L; normal, 14 to 40), and alanine aminotransferase (16 U/L; normal, 9 to 45), and complete blood count profiles were within the normal ranges. Urinalysis revealed a 4+ glucose level, but no ketones. Moreover, no sign of ketoacidosis was noted. The patient was screened for pancreatic autoantibodies (antibodies against glutamic acid decarboxylase, islet cell, islet antigen-2, and insulin) to rule out the possibility of type 1 diabetes, and tested negative. Liver and pancreas ultrasonography revealed no structural abnormalities.

The initial clinical management of the patient was continuous intravenous administration of short-acting insulin (1.5 IU/day; Humulin R, Lilly Pharmaceutical, Indianapolis, IN, USA). The insulin infusion rate was transiently increased up to $3.9 \mathrm{IU} /$ day for 0.5 hour at the start of every breastfeeding session. To monitor the glucose level and prevent hypoglycemia, fixed feeding inter vals of 2.5 to 3.0 hours were recommended and blood glucose was measured using heel prick blood sampling at the commencement of each feeding session. Ultimately, $2.4 \mathrm{IU} /$ day of insulin was required to maintain the blood glucose level in the range of 240 to $300 \mathrm{mg} / \mathrm{dL}$. While awaiting the results of genetic testing, transition to oral sulfonylurea (SU) $(0.2 \mathrm{mg} / \mathrm{kg} /$ day with gradual daily increases to $1.0 \mathrm{mg} / \mathrm{kg} /$ day while the insulin dose was decreased by $50 \%$ per day) was attempted to minimize potential neurodevelopmental disabilities associated with mutated ATPsensitive potassium (K-ATP) channels ${ }^{1)}$. The patient reverted to a hyperglycemic state. The SU trial treatment was discontinued 6 days after initiation, and the patient was switched back to intravenous insulin therapy, which resulted in a gradual improvement of the glycemic outcome. To facilitate continuation of treatment upon discharge, the transition from intravenous to subcutaneous insulin (MiniMed 640G, Medtronic, Minneapolis, MN, USA) administration was made 37 days postpartum. The pump was set to deliver a basal insulin amount of $3.0 \mathrm{IU} /$ day, with an additional 0.2 to $0.5 \mathrm{IU}$ of prefeeding bolus (preset square for 0.5 hour), active insulin time of 2.0 hours, predictive low-glucose suspend at $80 \mathrm{mg} / \mathrm{mL}$, and high glucose alert at $400 \mathrm{mg} / \mathrm{dL}$. The capabilities of the real-time CGM system in the latest model of the CSII pump are useful in eliminating heel pricks and episodes of hyperglycemia and hypoglycemia. The therapy was well tolerated, and the patient was discharged with the real-time CGM device and CSII pump on postnatal day 40 with an improved glycemic state and improved levels of C-peptide and insulin (Table 1, Figure 1). Upon discharge, his body weight was $5.0 \mathrm{~kg}$ (75th centile) and his length was $56 \mathrm{~cm}$ (75th to 90 th centile $)^{5)}$. The 
mean sensor glucose (MSG), the direct interstitial fluid glucose level measured by the CGM device, was $203 \pm 67 \mathrm{mg} / \mathrm{dL}$ until the age of 3 months, with a total daily insulin requirement of $3.8 \pm 0.9$ IU/day (Figure 2A). Subsequently, insulin therapy was no longer required and discontinued after 107 days. At the 4-month followup, the patient's body weight was $7.6 \mathrm{~kg}$ (90th to 95th centile) and his length was $66 \mathrm{~cm}$ (90th centile). The patient reached normal 4-month developmental milestones (laughing aloud and loss of head lag), with no observed neurodevelopmental impairment.

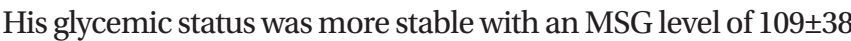
$\mathrm{mg} / \mathrm{dL}$ (Figure 2B). Ultrasonography of the liver and pancreas was repeated, which showed no abnormal findings. In addition, the patient experienced neither skin troubles associated with subcutaneous devices nor hyperglycemic or hypoglycemic episodes, and his glycated hemoglobin level was 5.7\% at age 6 months.

Genetic analysis was performed by isolating genomic DNA using a DNA sample preparation kit (TruSeq, Illumina, San Diego, CA, USA) and amplifying the enriched fragments of the target regions in the sequencing system (HiSeq2000, Illumina). The detection of insertions and deletions was based on an assigned algorithm (GATK RealignerTargetCreator, Broad Institute, Cam bridge, MA, USA), and base scores were recalibrated (GATK
BaseRecalibrator, Broad Institute). A targeted panel (ABCC8, BLK, CEL, eukaryotic translation initiation factor 2-alpha kinase 3 [EIF2AK3], FOXP3, GATA4, GATA6, GCK, HNF1A, HNF4A, HNF1B, INS, KCNJ11, KLF11, NEUROD1, PAX4, PDX1, pancreasassociated transcription factor 1-alpha [PTF1A], and ZFP57) sequencing for congenital diabetes identified two novel variants, as follows: a heterozygous missense variant (p.Arg1091Met, c.3272G $>$ T) in exon 17 of the EIF2AK3 gene and a heterozygous missense variant (p.Ser18Phe, c.53C $>\mathrm{T}$ ) in exon 1 of the $P T F 1 A$ gene, both of which are classified as variants of uncertain signifi cance (VUS) according to the American College of Medical Genetics guidelines $^{6}$. Sanger sequencing confirmed the presence of both variants. The patient's father was identified as a heterozygous carrier of the EIF2AK3 variant, whereas his mother was identified as a heterozygous carrier of the $P T F 1 A$ variant, indicating that the patient is a carrier and NDM was not triggered by autosomal recessive inheritance. Neither chromosomal microarray (CytoScan Dx assay, Affymetrix, ThermoFisher, Waltham, MA, USA) analysis nor methylation-specific multiple ligationdependent probe amplification detected relevant copy number changes or loss of methylation of $6 \mathrm{q} 24$.

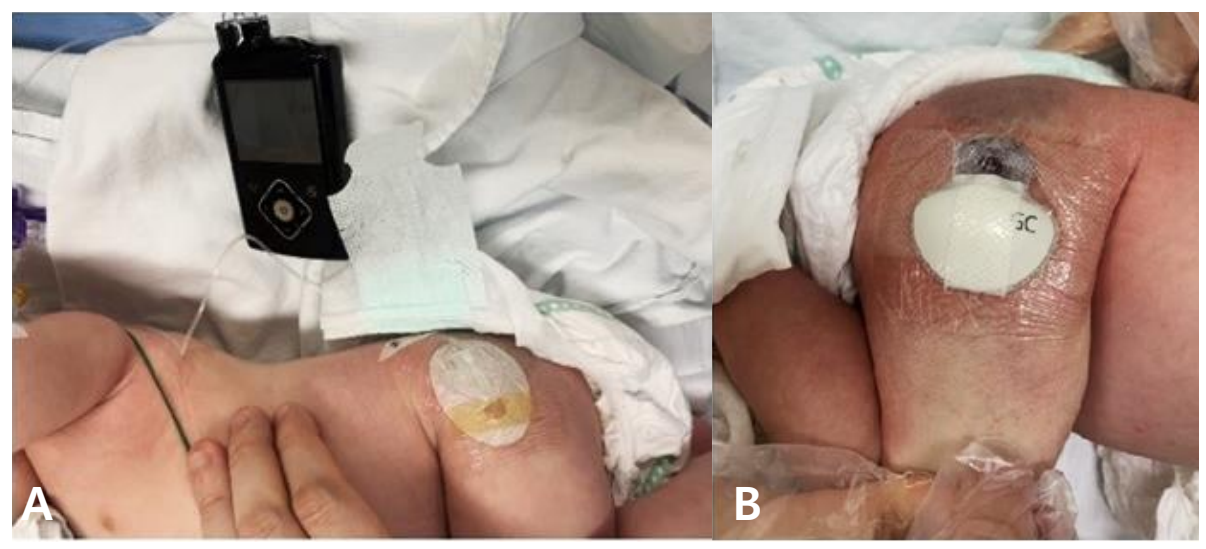

Figure 1. Successful transition to continuous subcutaneous insulin infusion (A) and realtime continuous glucose monitoring (B) on postpartum day 37 .

Table 1. Patient's Glucose, C-Peptide, and Insulin Trends during the Postnatal Period

\begin{tabular}{lcccc}
\hline & Postnatal day 7 & Postnatal day 20 & Postnatal day 40 & Postnatal day 220 \\
\hline Glucose $(\mathrm{mg} / \mathrm{dL})^{*}($ normal, 50-120) & 470 & 310 & 190 & 90 \\
C-peptide $(\mathrm{ng} / \mathrm{mL})^{*}($ normal, $0.4-3.3)$ & 0.06 & 0.13 & 0.31 & 1.00 \\
Insulin $(\mu \mathrm{U} / \mathrm{mL})^{*}$ (normal, $\left.0.0-2.5\right)$ & $<0.20$ & 0.34 & 0.67 & 1.78 \\
\hline
\end{tabular}

*All serum values were collected in the morning at the commencement of feeding. 


\section{DISCUSSION}

In neonates with hyperglycemia, NDM should not be immediately ruled out based on its low incidence rate. Impaired glucose homeostasis in the NICU can have numerous causes, including sepsis, stress, early protein intake, and use of various drugs $^{7)}$. Limited insulin secretion capacity, increased secretion of catecholamines induced by persistent hypoxia, and absence or delayed advancement of enteral feeding contribute to hyperglycemia and commonly occur in very preterm infants ${ }^{8)}$. Hyperglycemia in newborns normally occurs in the first 3 to 5 days after birth, and resolves within 2 or 3 days of onset; however, it may persist up to 10 days after birth ${ }^{1)}$. Although the clinical manifestations of dysglycemia in infants could be diverse, the major indication in our patient was shortness of breath, which necessitated the measurement of blood glucose level. Typical clinical signs such as polyuria, polydipsia, weight loss, or growth retardation may not always manifest in the presence of hyperglycemia. Therefore, it is crucial to examine the blood glucose level of any newborn who is acutely ill. As the level of glycated hemo globin could remain low until the age of 6 months (when hemo- globin A begins to replace fetal hemoglobin), it is not useful as a marker in the assessment of infantile $\mathrm{NDM}^{9)}$.

Both CGM and CSII are products of advanced technology for managing diabetes in pediatric patients. CSII is the subcutaneous continuous administration of short-acting insulin and can safely deliver small dosages even on random feeding. It rapidly switches off when the risk of hypoglycemic episodes is anticipated. Nevertheless, inconsistencies of appetite and feeding schedule and frequent infection of the needle insertion sites are factors that pose difficulties in treating diabetes during the neonatal period ${ }^{10)}$. Current CGM devices measure the glucose level in interstitial fluid using an amperometric glucose oxidase needle sensor inserted into the patient's subcutaneous tissue ${ }^{11)}$. Although sensor glucose levels may substantially deviate from the serum levels, and evidence remains unavailable for the clinical benefit and outcome of using CGM devices in the neonatal group, CGM is the only practical option to reduce the stress associated with frequent blood collection. Decreasing the frequency of heel pricks and prompt sensors enabling alarms for hypoglycemic or hyperglycemic episodes allowed for a more effective management of our patient's blood glucose level by the parents and

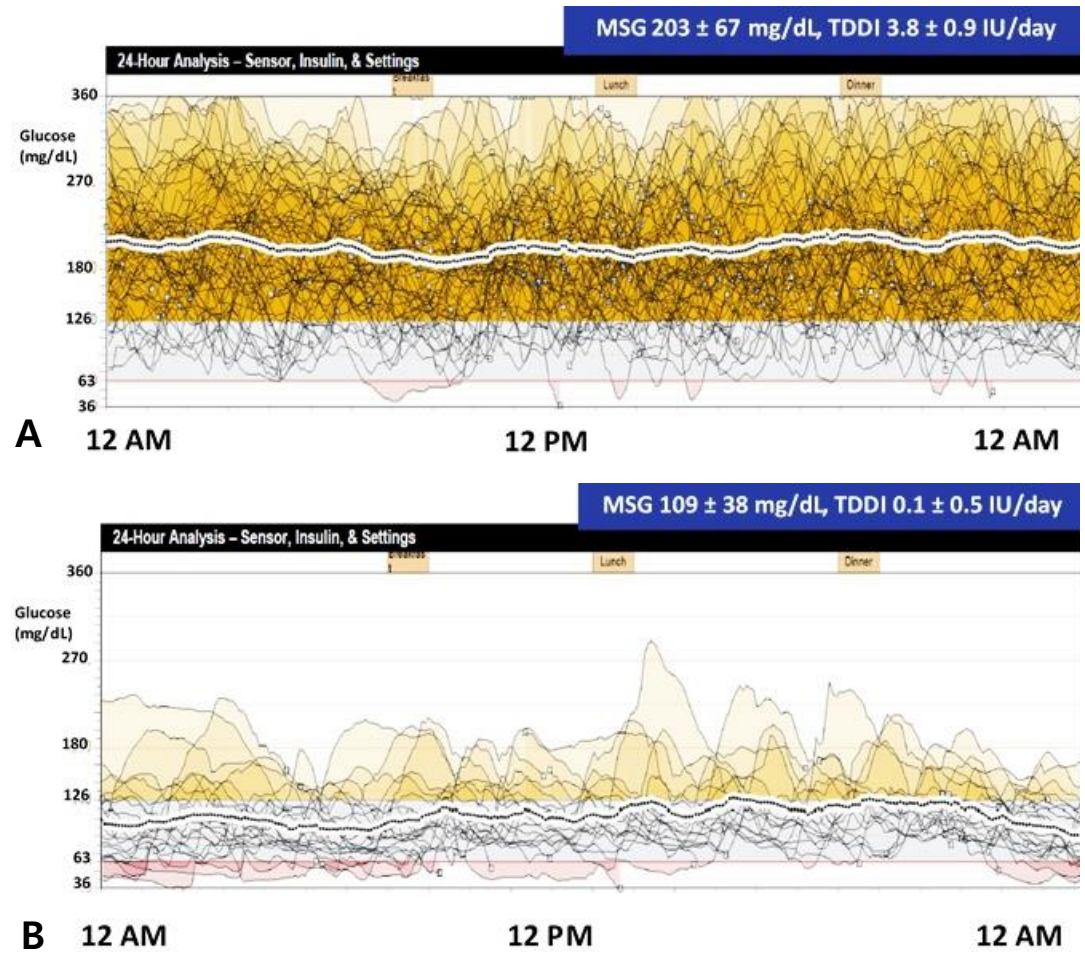

Figure 2. Twenty-four-hour analysis of mean sensor glucose (MSG) and total daily dose of insulin (TDDI) for the following periods: (A) age 1 to 3 months and (B) age 4 to 5 months. 
caregivers, thereby relieving the stress of glucose monitoring. The ideal insertion site for CGM devices is either the thigh or the upper buttock area with minimal subcutaneous fat ${ }^{1}$. Managing blood glucose levels in breastfeeding infants is especially challenging owing to frequent oral intake in variable quantities and repetitive preprandial delivery of small amounts of short-acting insulin.

In addition to CGM, although early insulin therapy at an initial dosage ranging from 0.02 to 0.05 units $/ \mathrm{kg} / \mathrm{hr}$ is widely used, the dosing should be adjusted based on the discretion of the attending clinician ${ }^{1)}$. To minimize hypoglycemic episodes, current technology offers a minimally invasive real-time monitoring of blood glucose and continuous delivery of insulin at a rate of as low as 0.025 units/hr. However, its long-term clinical efficacy and safety should be investigated in future studies. A potential limitation of the present case is that the recurrence of diabetes remains unknown, as long-term follow-up is not yet available.

Similar to maturity-onset diabetes of the young, NDM is typically known as a monogenic form of diabetes with highly penetrant inherited mutations in a single gene associated with $\beta$-cell function. Advancements in molecular technology have led to the identification of $80 \%$ of the etiologic genes of $\mathrm{NDM}^{1)}$. Nevertheless, the underlying genetic mechanism responsible for NDM, the basic physiology of glucose homeostasis, and the individual roles and interactions of each gene are yet to be fully understood. Recent studies have reported NDM as a polygenic disease with polygenic inheritance, and a diagnosis of diabetes in a child whose parents carry the mutations should not be excluded ${ }^{12)}$. Our patient presented with an overt clinical course of TNDM that required insulin therapy for slightly longer than 3 months.

Among the subgroups of NDM, TNDM is characterized by selflimited hyperglycemia, which lasts for a median of 12 weeks and completely resolves by the age of 18 months ${ }^{13,14)}$. TNDM accounts for approximately $45 \%$ of all NDM cases, PNDM accounts for most of the remaining 55\%, and syndromic NDM constitutes $<10 \%{ }^{10,15)}$. Overexpression of the imprinted genes at chromosome $6 \mathrm{q} 24$ as well as mutations in $Z F P 57$ and $H N F 1 B$ have been re ported to be responsible for TNDM. Defects in $K C N J 11, A B C C 8$, INS, and SLC2A2 can trigger either TNDM or PNDM ${ }^{1,16)}$. Despite the ultimate cessation of exogenous insulin treatment in TNDM, the probability rate of relapse and progression to PNDM is as high as $50 \%{ }^{3)}$. Intrauterine growth retardation is commonly observed in case of TNDM because insulin acts as a fetal growth hormone, and, consequently, its insufficiency coupled with failure of trans. placental insulin delivery causes newborns with TNDM to be born small for gestational age ${ }^{17,18)}$.

EIF2AK3, which is found on chromosome 2p11.2, encodes a protein kinase R-like endoplasmic reticulum kinase that is responsible for $\beta$-cell development ${ }^{19)}$. Its homozygous mutation is a common cause of Wolcott-Rallison syndrome, with clinical features including PNDM, multiple epiphyseal dysphasia, and growth retardation. In contrast, $P T F 1 A$, which is found on chromosome 10p12.2, encodes the PTF1A protein involved in pancreatic development. Its coding mutations cause agenesis of the pancreas, resulting in insufficient secretion of insulin, thereby causing diabetes mellitus in early postnatal life ${ }^{20)}$. Both $E I F 2 A K 3$ and PTF1A mutations are associated with PNDM and sponta neously arise with autosomal recessive inheritance ${ }^{1)}$. Our patient showed no clinical features associated with either WolcottRallison syndrome or pancreatic agenesis, and neither of his parents were diabetic. Consequently, the patient is expected to be a heterozygous carrier, indicating that the identified EIF2AK3 and $P T F 1 A$ variants were possibly not related to NDM pathogenicity. Nevertheless, it should be highlighted that various factors, in cluding intrauterine environment, perinatal stress, and diverse genetic background, could cause TNDM and require prompt management. Drawing a solid conclusion based on the genetic alterations identified in our patient was difficult because the pathogenicity is VUS limited and other causative genes of TNDM were not evaluated. When a variant is novel, poorly characterized, or controversial in terms of pathogenicity, it is classified as VUS, and VUS should not be used in clinical decision making because it provides insufficient information for determining pathogenicity ${ }^{6,21)}$. Therefore, additional functional studies and wider genetic screening would allow better interpretation and clarification of the effect of the identified variants on our patient.

Lastly, switching from insulin to oral SU was a substantial alternative even if the patient's glycemic control was deemed unfavorable during the transition period. Oral SU therapy permits insulin secretion through an ATP-independent closure of overly active mutated K-ATP channels. Thus, SU can rapidly ameliorate hyperglycemia in K-ATP channel-associated NDM, which accounts for $>50 \%$ of all cases of $\mathrm{NDM}^{1)}$. Early SU therapy may improve speech, motor, and cognitive disabilities, and recent studies have identified a higher prevalence of neurodevelop mental disorders in particular genotypes, although the correlation of the genotypes with neurodevelopmental impairment along with underlying pathophysiology remains to be charac 
terized $^{22,23)}$. Although variations of genes encoding the K-ATP channel are known to be the most common and second most common causes of PNDM and TNDM, respectively, genetic confirmation requires a considerable amount of time; however, recent literature suggests considering early SU therapy before a genetic diagnosis is made, to avoid the potential adverse effects of delayed therapy ${ }^{1,24)}$. The poor response to SU lowered the possibility of our patient having K-ATP channel mutation-related NDM.

In conclusion, our case suggests that consistent monitoring of blood glucose and genetic testing should be promptly performed in any newborns with persistent hyperglycemia, to prevent further diabetic complications. To date, CSII with CGM is the most effective and practical management for NDM. As the underlying causes of NDM are not fully understood, functional studies and wider genetic screening are needed for identifying and better understanding NDM-causing variants.

\section{ARTICLE INFORMATION}

\section{Ethical statement}

This study was approved by the Institutional Review Board of Seoul St. Mary's Hospital (IRB approval no.: KC20ZISI0264). Written informed consent was obtained from the participant's legal guardians/next of kin for the publication of this case report (including all data and images).

\section{Conflicts of interest}

No potential conflict of interest relevant to this article was reported.

\section{Author contributions}

Conception or design: M.S.K., M.B.A.

Acquisition, analysis, or interpretation of data: M.S.K., S.E.K., N.Y.L., S.K.K., S.H.K., W.K.C., K.S.C., M.B.A.

Drafting the work or revising: M.S.K., M.H.J., M.B.A.

Final approval of the manuscript: M.S.K., M.H.J., B.K.S., M.B.A.

\section{ORCID}

Min Soo Kim https://orcid.org/0000-0002-5549-3581

Moon Bae Ahn https://orcid.org/0000-0003-1108-2788

\section{Acknowledgments}

None

\section{REFERENCES}

1. Lemelman MB, Letourneau L, Greeley SAW. Neonatal diabetes mellitus: an update on diagnosis and management. Clin Perinatol 2018;45:41-59.

2. Sahebi L, Niknafs N, Dalili H, Amini E, Esmaeilnia T, Amoli M, et al. Iranian neonatal diabetes mellitus due to mutation in PDX1 gene: a case report. J Med Case Rep 2019;13:258.

3. Touati A, Errea-Dorronsoro J, Nouri S, Halleb Y, Pereda A, Ma hdhaoui $\mathrm{N}$, et al. Transient neonatal diabetes mellitus and hypomethylation at additional imprinted loci: novel ZFP57 mutation and review on the literature. Acta Diabetol 2019;56: 301-7.

4. Huang K, Liang L, Fu JF, Dong GP. Permanent neonatal diabetes mellitus in China. BMC Pediatr 2014;14:188.

5. im JS, Lim SW, Ahn JH, Song BS, Shim KS, Hwang IT. New Korean reference for birth weight by gestational age and sex: data from the Korean Statistical Information Service (2008-2012). Ann Pediatr Endocrinol Metab 2014;19:146-53.

6. Richards S, Aziz N, Bale S, Bick D, Das S, Gastier-Foster J, et al. Standards and guidelines for the interpretation of sequence variants: a joint consensus recommendation of the American College of Medical Genetics and Genomics and the Association for Molecular Pathology. Genet Med 2015;17:405-24.

7. Simsek DG, Ecevit A, Hatipoglu N, Coban A, Arisoy AE, Bas F, et al. Neonatal hyperglycemia, which threshold value, diagnostic approach and treatment?: Turkish Neonatal and Pediatric Endocrinology and Diabetes Societies consensus report. Turk Pediatri Ars 2018;53:S234-8.

8. Hay WW Jr, Rozance PJ. Neonatal hyperglycemia-causes, treatments, and cautions. J Pediatr 2018;200:6-8.

9. Suzuki S, Koga M, Niizeki N, Furuya A, Matsuo K, Tanahashi Y, et al. Evaluation of glycated hemoglobin and fetal hemoglobinadjusted HbAlc measurements in infants. Pediatr Diabetes 2013;14:267-72.

10. Kurnaz E, Aycan Z, Yildirim N, Cetinkaya S. Conventional insulin pump therapy in two neonatal diabetes patients harboring the homozygous PTF1A enhancer mutation: need for a novel approach for the management of neonatal diabetes. Turk J Pediatr 2017;59:458-62.

11. Shah R, McKinlay CJD, Harding JE. Neonatal hypoglycemia: continuous glucose monitoring. Curr Opin Pediatr 2018;30:204-

12. Cao B, Gong C, Wu D, Lu C, Liu F, Liu X, et al. Genetic analysis and follow-up of 25 neonatal diabetes mellitus patients in China. 
J Diabetes Res 2016;2016:6314368.

13. Naylor RN, Greeley SA, Bell GI, Philipson LH. Genetics and pathophysiology of neonatal diabetes mellitus. J Diabetes Investig 2011;2:158-69.

14. Loomba-Albrecht LA, Glaser NS, Styne DM, Bremer AA. An oral sulfonylurea in the treatment of transient neonatal diabetes mellitus. Clin Ther 2009;31:816-20.

15. Fu JL, WangT, XiaoXH. Relapsed 6q24-related transient neonatal diabetes mellitus successfully treated with sulfonylurea. Chin Med J(Engl) 2019;132:846-8.

16. Barbetti F, D'Annunzio G. Genetic causes and treatment of neonatal diabetes and early childhood diabetes. Best Pract Res Clin Endocrinol Metab 2018;32:575-91.

17. Aguilar-Bryan L, Bryan J. Neonatal diabetes mellitus. Endocr Rev 2008;29:265-91.

18. Rubio-Cabezas O, Ellard S. Diabetes mellitus in neonates and infants: genetic heterogeneity, clinical approach to diagnosis, and therapeutic options. Horm Res Paediatr 2013;80:137-46.

19. Asl SN, Vakili R, Vakili S, Soheilipour F, Hashemipour M, Ghahramani S, et al. Wolcott-Rallison syndrome in Iran: a common cause of neonatal diabetes. J Pediatr Endocrinol Metab 2019;32:
607-13.

20. Evliyaoglu O, Ercan O, Ataoglu E, Zubarioglu U, Ozcabi B, Dag deviren A, et al. Neonatal diabetes: two cases with isolated pancreas agenesis due to homozygous PTF1A enhancer mutations and one with developmental delay, epilepsy, and neonatal diabetes syndrome due to KCNJ11 mutation. J Clin Res Pediatr Endocrinol 2018;10:168-74.

21. Berg JS. Exploring the importance of case-level clinical information for variant interpretation. Genet Med 2017;19:3-5.

22. Shah RP, Spruyt K, Kragie BC, Greeley SA, Msall ME. Visuomotor performance in KCNJ11-related neonatal diabetes is impaired in children with DEND-associated mutations and may be improved by early treatment with sulfonylureas. Diabetes Care 2012;35:2086-8.

23. Svalastoga P, Sulen A, Fehn JR, Aukland SM, Irgens H, Sirnes E, et al. Intellectual disability in KATP channel neonatal diabetes. Diabetes Care 2020;43:526-33.

24. Li X, Xu A, Sheng H, Ting TH, Mao X, Huang X, et al. Early transition from insulin to sulfonylureas in neonatal diabetes and follow-up: experience from China. Pediatr Diabetes 2018;19:251-8. 